Caio Oliveira de Azevedo

\title{
Análise Dinâmica de Estilo na Recuperação das Exposições de Fundos de Investimentos Brasileiros: Uma Aplicação do Filtro de Kalman Restrito
}

Dissertação de Mestrado

Dissertação apresentada como requisito parcial para obtenção do título de Mestre pelo Programa de Pós-Graduação em Ciências Atuariais do Instituto de Gestão de Riscos Financeiros e Atuariais da PUC-Rio.

Orientador: Prof. Luciano Vereda Oliveira Co-Orientador: Prof. Cristiano Augusto Coelho Fernandes 


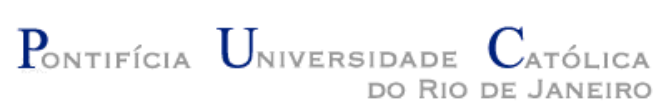

Caio Oliveira de Azevedo

\section{Análise Dinâmica de Estilo na Recuperação das Exposições de Fundos de Investimentos Brasileiros: Uma Aplicação do Filtro de Kalman Restrito}

Dissertação apresentada como requisito parcial para obtenção do título de Mestre pelo Programa de PósGraduação em Ciências Atuariais do Instituto de Gestão de Riscos Financeiros e Atuariais da PUC-Rio. Aprovada pela Comissão Examinadora abaixo assinada.

Prof. Luciano Vereda Oliveira Orientador Instituto de Gestão de Riscos Financeiros e Atuariais - PUC-Rio

Prof. Cristiano Augusto Coelho Fernandes Co-orientador Departamento de Engenharia Elétrica - PUC-Rio

Prof. Adrian Heringer Pizzinga Departamento de Engenharia Elétrica - PUC-Rio

Prof. Paulo Henrique Soto Costa Departamento de Engenharia Industrial - PUC-Rio

Prof. João Nizar Messari Coordenador(a) Setorial do Centro de Ciências Sociais - PUC-Rio 
Todos os direitos reservados. É proibida a reprodução total ou parcial do trabalho sem autorização da universidade, do autor e do orientador.

\section{Caio Oliveira de Azevedo}

Graduou-se como Bacharel em Ciências Econômicas pela Pontifícia Universidade Católica do Rio de Janeiro em 2005.

Ficha Catalográfica

Azevedo, Caio Oliveira de

Análise dinâmica de estilo na recuperação das exposições de fundos de investimentos brasileiros: uma aplicação do Filtro de Kalman restrito / Caio Oliveira de Azevedo; orientador: Luciano Vereda Oliveira; coorientador: Cristiano Augusto Coelho Fernandes - 2009.

119 f. ; $30 \mathrm{~cm}$

Dissertação (Mestrado em Gestão de Riscos Financeiros e Atuariais)-Pontifícia Universidade Católica do Rio de Janeiro, Rio de Janeiro, 2009.

Inclui bibliografia

1. Gestão de Riscos Financeiros e Atuariais Teses. 2. Análise dinâmica de estilo. 3. Modelos em espaço de estado lineares. 4. Filtro de Kalman. 5. Inicialização exata. 6. Restrições lineares. I. Oliveira, Luciano Vereda. II. Fernandes, Cristiano Augusto Coelho. III. Pontifícia Universidade Católica do Rio de Janeiro. Instituto de Gestão de Riscos Financeiros e Atuariais. IV. Título.

CDD: 368.01 
À Vida por ter me dado muita Luz para ver e Amor e Alegria para distribuir! 


\section{Agradecimentos}

Primeiramente, sou eternamente grato à Vida pelas experiências maravilhosas que pude compartilhar nestes meus 26 anos.

Meus agradecimentos sinceros aos meus pais, que compartilharam toda essa experiência de perto e, muitas vezes, colaboraram amorosamente.

Dedico também meus agradecimentos à Pontifícia Universidade Católica do Rio de Janeiro (PUC-Rio), à Escola Nacional de Seguros (Funenseg) e ao professor Luiz Roberto Cunha pelo provimento de toda a estrutura, inclusive financeira, que me permitiu hoje ter maior conhecimento em Atuária e Estatística.

Ao Instituto de Gestão de Riscos Financeiros e Atuariais da Pontifícia Universidade Católica do Rio de Janeiro (IAPUC) por acreditar no meu trabalho e me proporcionar aprendizados que carregarei sempre comigo. Dedico também meus agradecimentos à maravilhosa Lulu, por toda a sua dedicação e companheirismo.

Obviamente, dedico meus agradecimentos ao Luciano Vereda, meu orientador, e ao Cristiano Fernandes, meu co-orientador, que foram muito pacientes e solícitos, mesmo quando eu passava por momentos difíceis.

À minha lindona, que eu amo muito, que me ama muito, que esteve sempre do meu lado, dando-me inclusive uns "puxões" de orelha de vez em quando. 
À mamãe Sandra e toda a sua família pela sua dedicação, companheirismo e carinho.

Obviamente, não poderiam faltar meus queridos colegas de classe, que estão todos aqui presentes, sem exceção, mas que não precisam ser citados. Eles sabem que moram em meu coração.

Enfim, todos são importantes, cada qual com sua participação numa etapa da minha Vida. No entanto, tiveram duas pessoas que, de fato, foram especiais nestes momentos. Eu gostaria de ir além, afirmando que estas pessoas foram os verdadeiros artífices (artesanos) desta dissertação: o Adrian Pizzinga e o César Rivera.

Sem o curso de Espaço de Estado em suas fases teórica e operacionais, os permanentes incentivos, a dedicação quase que exclusiva quando ministrava suas aulas e a "garra" do Adrian em passar o conhecimento de que dispunha para nós, além de sua amizade, esta dissertação talvez nem tivesse ido à frente, ou mesmo nem teria um fundamento.

Quanto ao César, eu posso hoje dizer que sei programar em Matlab graças a ele. Dedico a ele meus sinceros votos de agradecimento, não pela ajuda técnica que me prestou somente, mas em especial pela sua grande amizade. Como escreveu uma vez minha namorada, "uma amizade que rompeu fronteiras e uniu dois países". 


\section{Resumo}

Azevedo, Caio Oliveira de; Vereda, Luciano (Orientador); Fernandes, Cristiano Augusto Coelho (Co-orientador). Análise Dinâmica de Estilo na Recuperação das Exposições de Fundos de Investimentos Brasileiros: Uma Aplicação do Filtro de Kalman Restrito. Rio de Janeiro, 2009. 119p. Dissertação de Mestrado - Instituto de Gestão de Riscos Financeiros e Atuariais, Pontifícia Universidade Católica do Rio de Janeiro.

Esta dissertação se propôs a investigar e interpretar o estilo de investimento de fundos de investimento brasileiros - fundos cambiais e de ações com administração ativa, no período de janeiro de 2004 a agosto de 2008, com o objetivo central de verificar se, de fato, estes fundos perseguem o estilo de investimento prometido aos seus clientes. Nesse sentido, foi empregada a metodologia de análise dinâmica de estilo baseada no retorno, utilizando o filtro de Kalman restrito reduzido com inicialização exata aplicado sobre modelos em espaço de estado. Para tanto, novos índices foram confeccionados a fim de cobrir a eventual carência de índices que pudessem apropriadamente representar as classes de ativos do mercado financeiro a que os fundos estão expostos. As principais conclusões obtidas foram: (1) mesmo em meio a um cenário adverso que marcou parte do período analisado, os fundos cambais mantiveram as estratégias de investimento anunciadas ao público, demonstrando transparência em suas ações; e (2) os fundos de ações estiveram, de fato, predominantemente expostos ao mercado bursátil, mas também alocaram parte considerável de seus recursos em títulos públicos federais de longo prazo indexados a índices de inflação.

\section{Palavras-chave}

Análise Dinâmica de Estilo; Modelos em Espaço de Estado Lineares; Filtro de Kalman; Inicialização Exata; Restrições Lineares. 


\section{Abstract}

Azevedo, Caio Oliveira de; Vereda, Luciano (Advisor); Fernandes, Cristiano Augusto Coelho (Co-advisor). Dynamic Style Analysis in Recovery of Brazilian Investment Funds Exposures: An Application of Restricted Kalman Filtering. Rio de Janeiro, 2009. 119p. MSc. Dissertation - Instituto de Gestão de Riscos Financeiros e Atuariais, Pontifícia Universidade Católica do Rio de Janeiro.

This dissertation aims to investigate and interpret the investment style of Brazilian investment funds - exchange funds and stock funds with active management, in the period ranging from January 2004 to August 2008, with the central objective of verifying if, in fact, these funds pursue the investment style promised to their customers. Accordingly, we used the methodology of returnbased dynamic style analysis, using the reduced restricted Kalman filtering with exact initialization applied to state space models. For this purpose, new indexes were created with the intention of covering the eventual lack of indexes that could appropriately represent the asset classes of financial market. The main conclusions were: (1) even in the midst of an adverse scenario that marked part of the analyzed period, the exchange funds kept the investment strategies announced to the public, demonstrating transparency in their actions; and (2) stock funds were indeed predominantly exposed to the stock market, but also allocated considerable part of their resources on federal public long-term bonds indexed by inflation indexes.

\section{Keywords}

Dynamic Style Analysis; Linear State Space Models; Kalman Filtering; Exact Initialization; Linear Restrictions. 


\section{Sumário}

1 Introdução

2 Modelos em Espaço de Estado 17

$\begin{array}{ll}2.1 \text { Motivação } & 17\end{array}$

2.2 Representação em Espaço de Estado Linear: Definição 18

3 Filtro de Kalman $\quad 20$

3.1 Os Três Enfoques: Predição, Filtragem e Suavização 20

3.1.1 Filtro de Kalman: Previsão e Atualização 22

3.1.2 Filtro de Kalman: Suavização 24

3.2 Inicialização do Filtro de Kalman $\quad 27$

3.2.1 Inicialização Difusa Aproximada 28

3.2.2 Inicialização Difusa Exata 29

3.3 Imposição de Restrições ao Filtro de Kalman 32

3.3.1 Filtro de Kalman Restrito Aumentado 33

3.3.2 Filtro de Kalman Restrito Reduzido 34

3.4 Estimação de Parâmetros por (Quasi) Máxima Verossimilhança 36

3.4.1 Estimação por Máxima Verossimilhança Não-Difusa 38

3.4.2 Estimação por Máxima Verossimilhança Difusa 39

3.5 Diagnósticos e Seleção de Modelos 40

4 Análise de Estilo 43

5 Base de Dados $\quad 47$

5.1 Fundos de Investimento Brasileiros $\quad 47$

5.1.1 Descrição dos fundos $\quad 47$

5.1.2 Análise das estatísticas descritivas dos retornos dos fundos $\quad 50$ 
5.2 Índices $\quad 53$

5.2.1 Descrição dos Índices 53

5.2.2 Confecção dos Quanta 56

5.2.2.1 Quantum Cambial 57

5.2.2.2 Quantum Prefixado 61

5.2.2.3 Quantum IGP-M 62

5.2.3 Análise dos retornos dos Índices 64

6 Análise de Estilo: Aplicações do Filtro de Kalman 77

6.1 Análise Dinâmica de Estilo 77

6.2 Modelagem e Interpretação dos Resultados $\quad 80$

6.2.1 Aspectos Principais da Modelagem 80

6.2.2 Modelagem final e Interpretação das Exposições Estimadas 82

6.2.2.1 A Economia Brasileira em 2004-2008: Uma Breve Análise 82

6.2.2.2 Estimação e Interpretação das Exposições 97

7 Conclusão 114

8 Referências Bibliográficas 116 


\section{Lista de figuras}

Figura 1 - Retornos dos fundos de ações ativo 50

Figura 2 - Retornos dos fundos cambiais dólar 51

Figura 3 - Retornos semanais dos índices 67

Figura 4 - Setor externo em milhões de US\$ 83

Figura 5 - Investimentos em carteira e IED em milhões de US\$ $\quad 86$

Figura 6 - Evolução das reservas internacionais e do câmbio 88

Figura 7 - Evolução das taxas de cupom cambial 91

Figura 8 - Evolução da Selic e das taxas internas de juros futuras $\quad 95$

Figura 9 - Evolução do Índice lbovespa 96

Figura 10 - Exposições suavizadas para o BB Ações

com respectivos intervalos de 95\% de confiança 102

Figura 11 - Diagnósticos gráficos sobre as inovações

padronizadas para o BB Ações 102

Figura 12 - Exposições suavizadas para o Itaú Ações

com respectivos intervalos de $95 \%$ de confiança

107

Figura 13 - Diagnósticos gráficos sobre as inovações

padronizadas para o Itaú Ações

107

Figura 14 - Exposições suavizadas para o Itaú Cambial

com respectivos intervalos de $95 \%$ de confiança

Figura 15 - Diagnósticos gráficos sobre as inovações

padronizadas para o Itaú Cambial

Figura 16 - Exposições suavizadas para o Bradesco Cambial

com respectivos intervalos de $95 \%$ de confiança

Figura 17 - Diagnósticos gráficos sobre as inovações

padronizadas para o Bradesco Cambial 


\section{Lista de tabelas}

Tabela 1 - Estatística descritiva dos retornos dos fundos 51

Tabela 2 - Matriz de correlação entre os retornos dos fundos 53

Tabela 3 - Estatísticas descritivas dos retornos dos índices (1 $1^{\underline{a}}$ parte) 71

Tabela 4 - Estatísticas descritivas dos retornos dos índices (2 $2^{\mathrm{a}}$ parte) 72

Tabela 5 - Matriz de correlação dos retornos dos índices (1 ${ }^{\underline{a}}$ parte) $\quad 75$

Tabela 6 - Matriz de correlação dos retornos dos índices ( $2^{\mathrm{a}}$ parte) $\quad 76$

Tabela 7 - Matriz de correlação dos retornos

dos fundos $\mathrm{x}$ retornos dos índices 99

Tabela 8 - Informações analíticas do modelo final para cada fundo 103 